\title{
Development of high-growth influenza H7N9 prepandemic candidate vaccine viruses in suspension MDCK cells
}

Tsai-Teng Tzeng ${ }^{1 \dagger}$, Po-Ling Chen ${ }^{1,2+}$, Tsai-Chuan Weng ${ }^{1}$, Shin-Yi Tsai ${ }^{1}$, Chia-Chun Lai ${ }^{1,3}$, Hsin-I Chou', Pin-Wen Chen ${ }^{1}$, Chia-Chun Lu', Ming-Tsan Liư ${ }^{4}$ Wang-Chou Sung ${ }^{1}$, Min-Shi Lee ${ }^{1}$ and Alan Yung-Chih Hu*

\begin{abstract}
Background: Influenza vaccine manufacturers traditionally use egg-derived candidate vaccine viruses (CWs) to produce high-yield influenza viruses for seasonal or pandemic vaccines; however, these egg-derived CWs need an adaptation process for the virus to grow in mammalian cells. The low yields of cell-based manufacturing systems using egg-derived CWs remain an unsolved issue. This study aimed to develop high-growth cell-derived CWs for MDCK cell-based vaccine manufacturing platforms.

Methods: Four H7N9 CWs were generated in characterized Vero and adherent MDCK (aMDCK) cells. Furthermore, reassortant viruses were amplified in adherent MDCK (aMDCK) cells with certification, and their growth characteristics were detected in aMDCK cells and new suspension MDCK (sMDCK) cells. Finally, the plaque-forming ability, biosafety, and immunogenicity of H7N9 reassortant viruses were evaluated.

Results: The HA titers of these CWs produced in proprietary suspension MDCK (sMDCK) cells and chicken embryos were 2- to 8-fold higher than those in aMDCK cells. All H7N9 CWs showed attenuated characteristics by trypsindependent plaque assay and chicken embryo lethality test. The alum-adjuvanted NHRI-RG5 (derived from the fifth wave H7N9 virus A/Guangdong/SP440/2017) vaccine had the highest immunogenicity and cross-reactivity among the four H7N9 CWs. Finally, we found that AddaVax adjuvant improved the cross-reactivity of low pathogenic H7N9 virus against highly pathogenic H7N9 viruses.

Conclusions: Our study indicates that cell-derived H7N9 CWs possessed high growth rate in new sMDCK cells and low pathogenicity in chicken embryo, and that CWs generated by this platform are also suitable for both cell- and egg-based prepandemic vaccine production.
\end{abstract}

Keywords: H7N9, Highly pathogenic avian influenza, Candidate vaccine virus, Suspension MDCK cells, Chemically defined medium, Reverse genetics

\footnotetext{
* Correspondence: alanhu@nhri.edu.tw

${ }^{\dagger}$ Tsai-Teng Tzeng and Po-Ling Chen contributed equally to this work.

'National Institute of Infectious Diseases and Vaccinology, National Health

Research Institutes (NHRI), 35 Keyan Road, Zhunan, Miaoli County 35053,

Taiwan

Full list of author information is available at the end of the article
}

(c) The Author(s). 2020 Open Access This article is licensed under a Creative Commons Attribution 4.0 International License, which permits use, sharing, adaptation, distribution and reproduction in any medium or format, as long as you give appropriate credit to the original author(s) and the source, provide a link to the Creative Commons licence, and indicate if changes were made. The images or other third party material in this article are included in the article's Creative Commons licence, unless indicated otherwise in a credit line to the material. If material is not included in the article's Creative Commons licence and your intended use is not permitted by statutory regulation or exceeds the permitted use, you will need to obtain permission directly from the copyright holder. To view a copy of this licence, visit http://creativecommons.org/licenses/by/4.0/ The Creative Commons Public Domain Dedication waiver (http://creativecommons.org/publicdomain/zero/1.0/) applies to the data made available in this article, unless otherwise stated in a credit line to the data. 


\section{Background}

The fifth epidemic wave of H7N9 avian influenza in China has raised concerns to public health. Up to May 2019, there have been 1568 human cases, including 616 deaths (mortality rate of approximately 39\%) [1]. Although the H7N9 outbreak is under control currently, the newly emerging H7N9 viruses still pose a threat to public health. The H7N9 viruses in the fifth wave have become highly pathogenic avian influenza (HPAI) viruses that bear mutations in the HA gene $[2,3]$ and display reduced susceptibility to antiviral drugs [4, 5]. Because of the close proximity of Taiwan to the H7N9 outbreak regions, it is crucial for the surveillance and control measure of poultry and migratory birds to be strengthened. Thus, antiviral medicines and H7N9 prepandemic vaccines need to be stockpiled in Taiwan.

Although egg-based manufacturing process is the most common platform to manufacture influenza vaccines in the past 70 years, this platform is labor-intensive and highly dependent on the supply of eggs. Compared with the egg-based production platform, cell-based production platform has more flexibility and scalability in manufacturing process and reduces the potential risk of egg shortage in a pandemic [6-8]. Moreover, cell-based influenza vaccines can avoid the potential risk of egg allergy [7]. Therefore, the cell-based production platform is an alternative manufacturing process to mitigate the shortage of influenza vaccine for pandemic preparedness.

Candidate vaccine viruses (CVVs) released from WHO Essential Regulatory Laboratories (ERLs) are usually derived from eggs and used to produce seasonal and pandemic influenza vaccines. However, the yield of egg-derived CVVs directly cultured in mammalian cells is usually low [9], which increases the cost of production and delays the supply of influenza vaccine during pandemics. To increase the yield of egg-derived CVVs in cell-based production platforms, further adaptation is usually required [10]. We produced inactivated whole-virion $\mathrm{H} 5 \mathrm{~N} 1$ and $\mathrm{H} 7 \mathrm{~N} 9$ vaccines in characterized adherent MDCK (aMDCK) cells in a PIC/ $\mathrm{S}$ GMP bioproduction plant at the National Health Research Institutes (NHRI), Taiwan [11, 12]. However, it took weeks to import the CVVs from ERLs and adapt the CVVs to an aMDCK cell-based platform before vaccine production. For a prompt response to an influenza pandemic, the preparation of cell-derived CVVs is desirable for the rapid production of cell-based pandemic influenza vaccines.

For the preparation of pandemic influenza CVVs, reverse genetic technology is a common method to generate high-growth reassortant viruses using six internal genes derived from A/Puerto Rico/8/34 (PR8) for eggbased manufacturing systems. However, the CVVs generated by using internal genes from PR8 are suitable for egg-based production platform. By using egg-derived CVVs in cell-based platforms, adaptation is usually needed, which takes extra time for preparing production. To shorten the preparation time of vaccine viruses for cell-based manufacturing systems, recent studies used cell-adapted high-growth viruses as master donor viruses (MDVs) [13-15], and synthetic hemagglutinin (HA) and neuraminidase (NA) genes [16, 17] to generate cellderived CVVs. In addition, for safety issue, the cleavage site of HA protein was modified to -PKGR-, a cleavage site of LPAI H7N9 viruses, and the position 292 of NA protein was also modified to Arginine ( $R$ ) to increase the sensitivity to NA inhibitor, such as oseltamivir and zanamivir [18]. In this study, we generated cell-derived H7N9 CVVs by reverse genetics (six internal genes derived from an aMDCK cell-adapted H5N1 virus (NIBRG-14) and two attenuated synthetic HA and NA genes). A strategy to enhance the virus yield of CVVs by using the cell-derived CVV technology in our proprietary suspension MDCK (NHRI sMDCK) cells was presented.

\section{Methods \\ Viruses, cells, and medium}

The A/Taiwan/1/2017 H7N9 wild-type virus was kindly supplied by the Taiwan CDC. Four H7N9 CVVs were generated using reverse genetics. Vero cells (ATCC CCL81) were cultivated in VP-SFM medium (GibcoBRL) supplemented with $4 \mathrm{mM}$ glutamine (GibcoBRL), and aMDCK cells (ATCC CCL-34) were cultivated in OptiPro medium (GibcoBRL) supplemented with $4 \mathrm{mM}$ glutamine. After adaptation to serum-free media, Vero and aMDCK cell banks were characterized by Bioreliance (UK), and both cell lines were confirmed to be nontumorigenic. For viral growth, the culture medium was supplemented with $2 \mu \mathrm{g} / \mathrm{mL}$ TPCK-trypsin (Sigma).

\section{Adaptation of adherent MDCK cells to suspension growth} The proprietary sMDCK cells were cultivated in chemically defined BalanCD ${ }^{\circ}$ Simple MDCK medium (FUJFILM Irvine Scientific) supplemented with $4 \mathrm{mM}$ glutamine, and the suspension adaptation process was previously shown in details (PCT Patent No. WO2017072744A1). The adherent MDCK cells were seeded at $5 \times 10^{5}$ cells $/ \mathrm{mL}$ in spinner flask without microcarriers and culture medium contained $5 \%(\mathrm{v} / \mathrm{v})$ fetal bovine serum (FBS). Spinner flasks were placed on a stir plate $(45-55 \mathrm{rpm})$ in a $37^{\circ} \mathrm{C}$, humidified incubator with $5 \% \mathrm{CO}_{2}$. During the period of adaptation, cells in the suspension culture was refreshed with commercial serum-free medium every 3-4 days until the cells grew in suspension (i.e. minimal aggregation) with low proportion of serum. In order to grow the suspension-adapted MDCK (sMDCK) cells without serum, the sMDCK cells were further adapted with several commercial serum-free medium and were frozen with $10 \%$ DMSO as a master cell stock. The sMDCK cells were finally adapted into serum-free BalanCD $^{\circ}$ Simple MDCK medium and were frozen with $10 \%$ 
DMSO as a working cell stock. For routine maintenance in spinner flask, sMDCK cells were inoculated at a concentration of $2 \times 10^{5}$ cells $/ \mathrm{mL}$ in serum-free BalanCD ${ }^{\circ}$ Simple MDCK medium, and maximum cell density in suspension cultures are about $1.8 \times 10^{6}$ cells $/ \mathrm{mL}$.

\section{Plasmid construction}

Six internal genes derived from aMDCK-adapted NIBRG14 virus were reverse transcribed using Uni12 primer and amplified with a universal primer set [19]. The HA and NA genes of H7N9 viruses were synthesized (GENEWIZ), and the polybasic cleavage site in HA and position 292 (N2 numbering) of the NA gene were modified (see Table 1). Eight influenza virus genes were cloned into the pHW2000 vector for rescuing CVVs using reverse genetics [20].

\section{Generation of H7N9 CVVs by reverse genetics}

For safety, all experiments with H7N9 CVVs were conducted in biosafety level 3 (BSL-3) containment approved by the Taiwan CDC. To rescue the 6:2 CVVs, eight plasmids expressing HA, NA, and six internal genes were transfected into Vero cells by electroporation (Fig. 1B). At 4 days posttransfection, virus-containing supernatant (designated V1) was collected and added to aMDCK or sMDCK cells to amplify the rescued viruses (V1aM1 or V1sM1). Viral titers were determined by hemagglutination (HA) and $50 \%$ tissue culture infective dose $\left(\mathrm{TCID}_{50}\right)$ assays. The passage history of the reassortant viruses was labeled with the number of passages in the indicated cells (V, Vero cells; aM, adherent MDCK cells; sM, suspension MDCK cells; E, eggs). For example, V1aM3 indicates that the reassortant virus was initially grown in Vero cells, followed by 3 passages in aMDCK cells.

\section{Evaluation of viral growth properties}

A confluent monolayer of aMDCK cells (approximately $2 \times 10^{7}$ cells) was grown in a T150 flask and infected with reassortant viruses (V1aM3) at a multiplicity of infection (MOI) of 0.0001. Suspension MDCK cells were grown in a spinner flask. When the cell density of sMDCK cells reached approximately $1.8 \times 10^{6}$ cells $/ \mathrm{mL}$ from a seeding density of $2.5 \times 10^{5}$ cells $/ \mathrm{mL}$, reassortant viruses (V1aM3) were inoculated at an MOI of 0.0001. Finally, the supernatant was collected daily after infection.

\section{Analysis of genetic stability}

To evaluate the genetic stability of CVVs, the reassortant viruses (V1aM3 and V1sM3) were grown in MDCK cells for 4 passages. As mentioned before, the viruses were inoculated to MDCK cells and collected after incubation for $72 \mathrm{~h}$ at $35^{\circ} \mathrm{C}$, and the HA and NA genes of the virus stocks (V1aM3 and V1aM7) were sequenced.

\section{Plaque assay in MDCK cells with or without trypsin}

The plaque characteristics of the reassortant viruses (V1aM3) were determined on aMDCK cells. aMDCK cells were grown on 6-well culture plates. Before infection, aMDCK cells were washed with $1 \times$ Dulbecco's phosphatebuffered saline (DPBS) and inoculated with $0.5 \mathrm{~mL}$ of diluted viruses in each well. After $60 \mathrm{~min}$ of incubation at $35^{\circ} \mathrm{C}$, the cells were washed with $1 \times$ DPBS and covered with $3 \mathrm{~mL}$ of medium containing $0.3 \%$ agarose with or without $1 \mu \mathrm{g} / \mathrm{mL}$ TPCK-treated trypsin. After $72 \mathrm{~h}$ of incubation at $35^{\circ} \mathrm{C}$, the cells were fixed with $3.7 \%$ formaldehyde and stained with $0.5 \%$ crystal violet to visualize the plaques.

\section{Chicken embryo lethality test}

This study protocol was approved by the Institutional Animal Care and Use Committee of NHRI (Protocol No: NHRI-IACUC-107030-A). Ten-day-old chicken eggs (specific antibody-negative eggs) were inoculated with $0.1 \mathrm{~mL}$ of 10 -fold serially diluted virus (V1aM3) with known $\mathrm{TCID}_{50}$ titers. Embryo viability was recorded at $48 \mathrm{~h}$ postinfection. The median chicken embryo lethal dose $\left(C E L D_{50}\right)$ was calculated by the method of Reed and Muench [21]. Furthermore, the allantoic fluid of six eggs injected with viruses (V1aM3) diluted 1000-fold was harvested on the third day postinfection (DPI). The HA titer and viral titer of the harvested allantoic fluid were determined by hemagglutination and $\mathrm{TCID}_{50}$ assays, respectively.

\section{Purification for $\mathrm{H} 7 \mathrm{~N} 9$ reassortant viruses}

The H7N9 bulks produced in sMDCK cells were purified according to a previous study [22] with the following modifications. First, $400 \mathrm{~mL}$ of the harvested virus was inactivated, and then the cell debris was removed using centrifugation at $1800 \times \mathrm{g}$ for $30 \mathrm{~min}$. Next, the inactivated virus was further purified using Capto $Q$ and

Table 1 Hemagglutinin cleavage site and NAI resistance marker in wild-type viruses and CWs

\begin{tabular}{|c|c|c|c|c|c|c|}
\hline \multirow[t]{2}{*}{ CWs } & \multirow[t]{2}{*}{ Wild-type viruses } & \multirow[t]{2}{*}{ Pathogenicity } & \multicolumn{2}{|c|}{ Modification of hemagglutinin cleavage site } & \multicolumn{2}{|c|}{ Modification of NAI resistance marker (R292K) } \\
\hline & & & Wild type & CWs & Wild type & CWs \\
\hline NHRI-RG3 & A/Guangdong/17SF003/2016 & HPAl & PEVPKRKRTAR/GLF & PEVPKGR/GLF & K & R \\
\hline NHRI-RG4 & A/Hong Kong/125/2017 & LPAI & PEIPKGR/GLF & PEIPKGR/GLF & $\mathrm{R}$ & $\mathrm{R}$ \\
\hline NHRI-RG5 & A/Guangdong/SP440/2017 & HPAl & PEVPKRKRTAR/GLF & PEVPKGR/GLF & K & $\mathrm{R}$ \\
\hline NHRI-RG6 & A/Taiwan/1/2017 & HPAl & PEVPKRKRTAR/GLF & PEVPKGR/GLF & K & $R$ \\
\hline
\end{tabular}

Note: HPAl highly pathogenic avian influenza, LPAI low pathogenic avian influenza, NAl neuraminidase inhibitor 


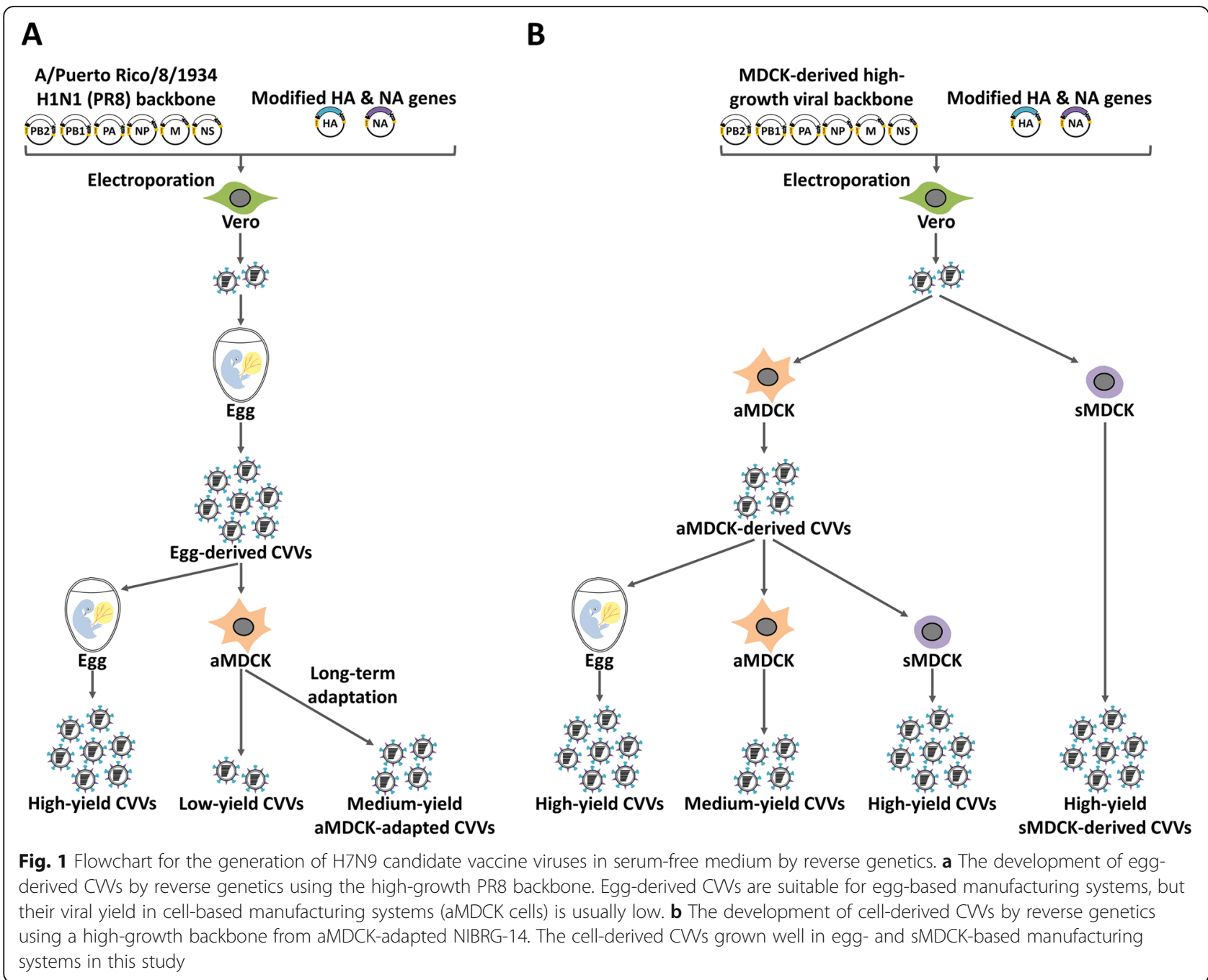

Capto core 700 anion exchange chromatography columns in an AKTA purifier 100 system (GE Healthcare). The flow-through virus solution was diafiltered with PBS using tangential flow filtration with a $100 \mathrm{kDa}$ membrane cassette (Sartorius). Finally, this purified virus bulk was sterilized by using a $0.22 \mu \mathrm{m}$ filter and stored at $4{ }^{\circ} \mathrm{C}$. The amount of HA antigen in the bulk virus stock was calculated based on the band intensity of the viral protein on a $10 \%$ NuPAGE Bis-Tris gel (Thermo Fisher Scientific), and the amount of total viral protein was measured using a Modified Lowry Protein Assay kit (Thermo Fisher Scientific) [23].

\section{Vaccine preparation and immunization}

The mouse study protocol was approved by the Institutional Animal Care and Use Committee of NHRI (Protocol No: NHRI-IACUC-107106-A). Six-week-old female $\mathrm{BALB} / \mathrm{c}$ mice ( $n=6$ per group) were intramuscularly injected with two doses of vaccine (at day 0 and day 14) containing $0.2 \mu \mathrm{g}$ of $\mathrm{HA}$ antigen and aluminum hydroxide (Brenntag AG) or AddaVax ${ }^{\mathrm{mat}}$ (InvivoGen) adjuvant. The amount of alum adjuvant was $30 \mu \mathrm{g}$ per dose. AddaVax was used according to the manufacturer's instructions. At day 28, blood samples were collected in serum separator tubes (BD BioSciences). The serum was isolated by centrifugation at $3000 \mathrm{rpm}$ for 10 min and stored at $-20^{\circ} \mathrm{C}$.

\section{Hemagglutination inhibition (HI) assay}

The hemagglutination inhibition (HI) assay was used to assess functional antibodies that inhibit hemagglutination [24]. The standard HA antigen (No. 18/196) and HA antiserum (No. 18/112) for H7N9 (A/Guangdong/17SF003/ 2016) influenza virus, were purchased from the UK NIBSC.

\section{Statistical analysis}

Statistical data were generated using GraphPad Prism 5 software. HI titers were transformed into logarithmic values and statistical significance between groups was 
analyzed by one-way analysis of variance (ANOVA) with Newman-Keuls post-test [25].

\section{Results}

Selection of H7N9 strains for CVV preparation

WHO ERLs usually developed egg-derived CVVs for influenza vaccine production. To evaluate the potential of our cell-derived CVVs, we selected two H7N9 viruses (A/ Guangdong/17SF003/2016 and A/Hong Kong/125/2017) that were used by WHO ERLs. Our previous study showed that the acquisition of glycosylation site in HA protein during the adaptation of the NIBRG-268 virus in MDCK cells improved the HA titer and growth efficiency of the influenza H7N9 virus without affecting viral antigenicity [12]. To explore the role of acquired glycosylation in the growth of H7N9 CVV in MDCK cells, we also generated CVVs derived from the A/Guangdong/SP440/2017 and A/Taiwan/1/2017 H7N9 viruses, which acquired potential $\mathrm{N}$-linked glycosylation site in HA protein during evolution. In this study, four H7N9 CVVs were prepared, and their growth rate, genetic stability, biosafety, and immunogenicity were analyzed.

\section{Growth property of H7N9 reassortant viruses}

To increase viral yields in the aMDCK-based production system, we generated cell-derived CVVs by reverse genetics using an aMDCK-adapted NIBRG-14 virus (an $\mathrm{H} 5 \mathrm{~N} 1$ candidate vaccine virus) an MDV. The HA titers of NHRI-RG5 and NHRI-RG6 in aMDCK cells ranged from 128 to 256 hemagglutination units (HAU)/50 $\mu \mathrm{L}$ (Table 2) and were maintained at a similar level after several passages (V1aM10, unpublished data). In contrast, the HA titers of NHRI-RG3 and NHRI-RG4 showed an increase from $64 \mathrm{HAU} / 50 \mu \mathrm{L}(\mathrm{V} 1 \mathrm{aM} 2)$ to $256 \mathrm{HAU} / 50 \mu \mathrm{L}(\mathrm{V} 1 \mathrm{aM} 3)$ and were maintained at a level similar to that of V1aM10 (unpublished data), which means in the aMDCK-based production system, the aMDCK cell-adapted MDV could not generate highgrowth reassortants for all H7N9 viruses and the reassortant viruses may need further adaptations to reach higher titers (Table 2).

Recently, we developed an sMDCK cell line to overcome the problem of scalability in the adherent cellbased manufacturing process for influenza vaccines. To explore the productivity of CVVs in sMDCK cells, the rescued reassortant viruses were also directly inoculated to the cells. The HA titers of sMDCK-derived NHRIRG3, NHRI-RG4 increased from $64 \mathrm{HAU} / 50 \mu \mathrm{L}$ (V1aM1) to 256/512 HAU/50 $\mu \mathrm{L}$ (V1sM1) (Table 2). Similarly, the HA titers of sMDCK-derived NHRI-RG5 and -RG6 showed an 8- to 16-fold increase, compared with their aMDCK-derived counterparts (Table 2). These results suggest that the inoculation of the rescued reassortant virus in sMDCK cells directly improved viral titer by 8 - to 16 -fold without further adaptation. Interestingly, the MDCK-derived reassortant viruses still have a good growth property in eggs, and could grow to high HA titers in chicken embryos which reaching 2048 HA/ $50 \mu \mathrm{L}$ (V1aM3E1, Table 2).

To evaluate the antigen yield of CVVs in aMDCK and sMDCK cells, we analyzed the viral growth kinetic of H7N9 CVVs. The H7N9 CVVs from sMDCK cells showed an 4-fold increase in HA titers when grown in sMDCK cells (the solid line in Fig. 2) compared with that from aMDCK cells. Similarly, the infectious titers of CVVs at day 1, 2, and 3 after infection were shown in Fig. 2 (dashed line), and the peak viral titers of each CVVs were observed by day 2. The infectious titers of CVVs in sMDCK cells were higher than that in aMDCK cells at day 2 after infection. Taken together, these data indicate that the sMDCK-based production platform has commercial potential for influenza vaccines.

\section{Genetic stability of the HA and NA genes in H7N9 CVVs}

To evaluate the genetic stability of the HA and NA genes, aMDCK and sMDCK cells were inoculated with H7N9 CVVs for multiple passages and the sequence of the HA and NA genes in passage 3 and 7 was analyzed. The NA sequences were not changed up to passage 7 and the introduced K292R in NA (Table 1) was retained. Importantly, the sequence of the modified HA cleavage site remained unchanged as a monobasic amino acid in passage 3 and 7, suggesting that H7N9 CVVs remained low pathogenic avian influenza (LPAI) up to passage 7.

As mentioned previously, there was an increase in the HA titers of NHRI-RG3 and NHRI-RG4 at the V1aM3 and V1sM3 passages (Table 2). The sequencing results showed that an HA-I120T mutation (H7 numbering)

Table 2 Viral titers of H7N9 CWs after serial passaging in Vero cells, MDCK cells, and chicken embryonic eggs

\begin{tabular}{|c|c|c|c|c|c|c|c|c|}
\hline \multirow[t]{2}{*}{ CWs } & \multicolumn{8}{|c|}{ HA titer $(\mathrm{HAU} / 50 \mu \mathrm{L})$ at each passage ${ }^{\mathrm{a}}$} \\
\hline & V1 & V1aM1 & V1aM2 & V1aM3 & V1aM3E1 & V1sM1 & $\mathrm{V} 1 \mathrm{sM} 2$ & V1sM3 \\
\hline NHRI-RG3 & 4 & 64 & 64 & 256 & 2048 & 512 & 512 & 1024 \\
\hline NHRI-RG4 & 8 & 64 & 64 & 256 & 2048 & 512 & 512 & 2048 \\
\hline NHRI-RG5 & 64 & 256 & 256 & 256 & 2048 & 2048 & 2048 & 2048 \\
\hline NHRI-RG6 & 64 & 128 & 256 & 256 & 2048 & 2048 & 2048 & 2048 \\
\hline
\end{tabular}

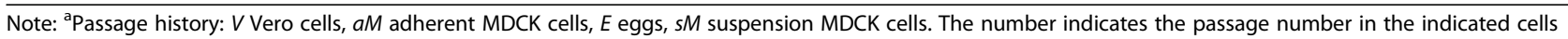




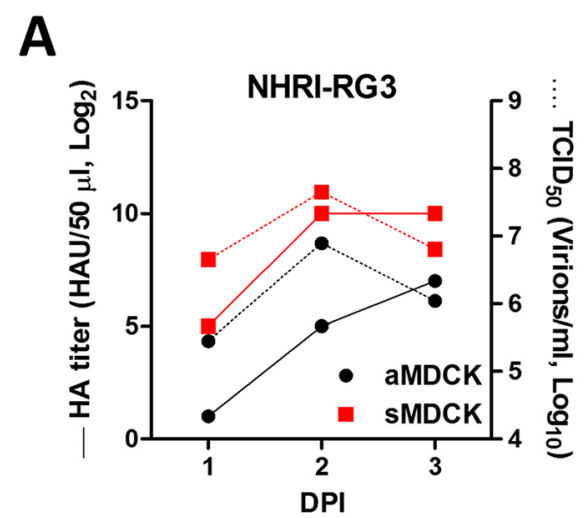

C

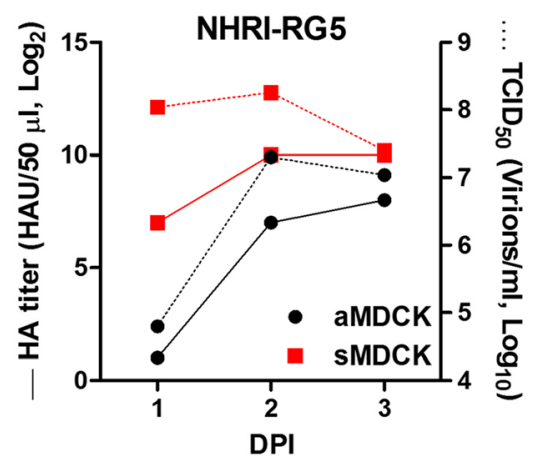

B

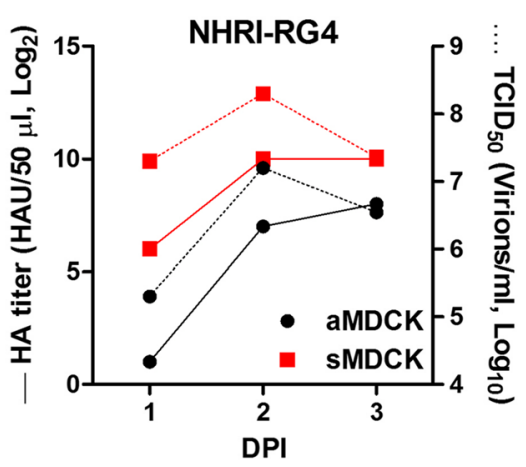

D

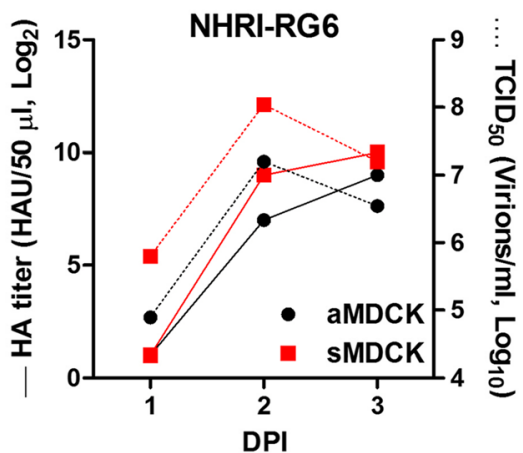

Fig. 2 Growth properties of reassortant H7N9 viruses in aMDCK and sMDCK cells. a NHRI-RG3 (A/Guangdong/17SF003/2016 H7N9), (b) NHRI-RG4 (A/Hong Kong/125/2017 H7N9), (c) NHRI-RG5 (A/Guangdong/SP440/2017 H7N9), and (d) NHRI-RG6 (A/Taiwan/1/2017 H7N9). Cells were infected with the indicated viruses (V1aM3) at a multiplicity of infection (MOI) of 0.0001 and incubated at $35^{\circ} \mathrm{C}$. Cell culture supernatants were collected at 1,2 , and 3 days postinfection (DPI). The HA titer (solid line) and viral titer (dashed line) of the collected supernatants were determined by hemagglutination and $\mathrm{TCID}_{50}$ assays, respectively

appeared in NHRI-RG3 during the V1aM3 passage, and this mutation became dominant at the V1aM7 passage (Table 3). There was also a mutation, HA-A151T, in NHRI-RG4 at the V1aM3 passage, and this mutation was maintained at the V1aM7 passage (Table 3). No mutation was identified in internal genes (PB1, PB2, PA, NP, NS, and M genes) of H7N9 CVVs propagated in aMDCK or sMDCK cells, hence, these HA mutations in NHRI-RG3 and NHRI-RG4 may be the cause of increased HA titer. In terms of NHRI-RG3 and NHRIRG4 at the V1sM3 and V1sM7 passage, several amino acid substitutions in HA were found in NHRI-RG3 (I120T, K163R, and A292T) and NHRI-RG4 (I120T,
I421V, and I421M) (Table 3). Interestingly, I120T substitution was found in NHRI-RG3 (V1aM3 and V1sM3) and NHRI-RG4 (V1sM4). Although the role of other substitutions remains unclear, I120T substitution could be the main factor for the improved viral growth of these viruses.

\section{Trypsin-dependent plaque formation}

Trypsin-dependent plaque formation is a feature of LPAI and attenuated CVVs. A previous study showed that HPAI H7N9 has a trypsin-independent plaque-forming ability [2], which is conferred by the polybasic cleavage site in HA. The modified HA cleavage site of H7N9

Table 3 Amino acid substitutions in the HA protein of H7N9 CWs propagated in aMDCK or sMDCK cells

\begin{tabular}{|c|c|c|c|c|}
\hline CWs & V1aM3 $^{a}$ & $\mathrm{~V} 1 \mathrm{aM}^{\mathrm{a}}$ & $\mathrm{V}_{1} \mathrm{sM}^{\mathrm{a}}$ & $\mathrm{V} 1 \mathrm{sM} 7^{\mathrm{a}}$ \\
\hline NHRI-RG3 & $1120 T /\left.\right|^{b}$ & 1120T & $|120| / T^{b}, G 209 E / G^{b}$ & I120T, K163R, A292T/A \\
\hline NHRI-RG4 & A151T & A151T & $1120 \mid / T^{\mathrm{b}}$ & $1120 T / /^{b}, 14211 / N / M^{b}$ \\
\hline NHRI-RG5 & No change & No change & No change & No change \\
\hline NHRI-RG6 & No change & No change & No change & No change \\
\hline
\end{tabular}

Note: ${ }^{\text {P}}$ Passage history: $V$ Vero cells, $a M$ adherent MDCK cells, $s M$ suspension MDCK cells. The number indicates the passage number in the indicated cells ${ }^{\mathrm{b}}$ Mixed amino acid residues were detected 
CVVs was similar to that of LPAI, so we examined whether the plaque-forming ability of H7N9 CVVs is trypsin-dependent. In the presence of trypsin, H7N9 CVVs exhibited viral titers ranging from $10^{8.20}$ to $10^{9.05}$ $\mathrm{PFU} / \mathrm{mL}$ (Table 4). In the absence of trypsin, the plaqueforming ability of H7N9 CVVs was reduced 10-fold (Table 4), and the plaque size was reduced (Fig. 3). These results indicate that the plaque-forming ability of all H7N9 CVVs is trypsin-dependent, which is consistent with the sequence of the monobasic cleavage site in HA.

\section{Biosafety evaluation by using chicken embryonic eggs}

Before vaccine manufacture, the biosafety of CVVs is usually evaluated in eggs, chickens, and ferrets [26]. Previous study showed that chicken embryo lethality of CVVs and HPAI viruses is consistent with their pathogenicity in chickens and ferrets [27]. Because our current facility is not equipped for experiments in chickens and ferrets, we tested the pathogenicity of H7N9 CVVs in chicken embryonic eggs. HPAI H7N9 virus (A/Taiwan/ 1/2017) was used as a positive control, and its $\operatorname{CELD}_{50}$ was $10^{-1.17} \mathrm{TCID}_{50}$ (Table 5). In contrast, the $\mathrm{CELD}_{50}$ of H7N9 CVVs in this study was more than $10^{6.65}$ TCID $_{50}$ (Table 5), suggesting that the CVVs were attenuated with low levels of pathogenicity.

\section{Antigenicity and immunogenicity of the 5th wave H7N9 vaccines}

The major differences in the HA1 sequences among the selected H7N9 CVVs included I38T, T112P, I120T, K130R, K164E, L217Q, and I317V (Additional file 1: Table S1). To investigate the antigenicity and immunogenicity of the CVVs, H7N9 CVVs were produced in sMDCK cells and purified by using flow-through chromatography. Electron microscopy images revealed that the four purified H7N9 CVVs had a spherical shape with clear spike structures on the surface (Additional file 2: Fig. S1). The antigenicity of H7N9 reassortant viruses was determined by $\mathrm{HI}$ assay using A/Guangdong/ 17SF003/2016-like standard serum. The HI assay results revealed that the antigenicity of NHRI-RG3, RG4, and RG5 (V1aM3sM1) was similar to that of A/Guangdong/ 17SF003/2016-like standard antigen (Table 6). The sequencing results showed that NHRI-RG3, RG4, and RG5 at V1aM3sM1 passage had HA-I120T or HA-A151T

Table 4 Plaque formation of H7N9 CWs

\begin{tabular}{lll}
\hline CWs & With trypsin & Without trypsin \\
\hline NHRI-RG3 & $10^{8.34} \mathrm{PFU} / \mathrm{mL}$ & $10^{7.07} \mathrm{PFU} / \mathrm{mL}$ \\
NHRI-RG4 & $10^{8.20} \mathrm{PFU} / \mathrm{mL}$ & $10^{7.45} \mathrm{PFU} / \mathrm{mL}$ \\
NHRI-RG5 & $10^{9.05} \mathrm{PFU} / \mathrm{mL}$ & $10^{8.10} \mathrm{PFU} / \mathrm{mL}$ \\
NHRI-RG6 & $10^{8.50} \mathrm{PFU} / \mathrm{mL}$ & $10^{7.22} \mathrm{PFU} / \mathrm{mL}$ \\
\hline
\end{tabular}

Note: PFU plaque-forming units substitutes without other mutation after an sMDCK passage (unpublished data). Hence, these results indicated that HA-I120T and HA-A151T substitutes did not change viral antigenicity. Notably, the HI titer of NHRIRG6 was 4-fold lower than that of NHRI-RG3, RG4, and RG5, as well as standard antigen, suggesting that the antigenicity of NHRI-RG6 was different with those viruses (Table 6). NHRI-RG6 (derived from A/Taiwan/1/2017) had several differences in HA1 amino acid sequence compared with A/Guangdong/17SF003/2016 H7N9 virus (Additional file 1: Table S1), which could be the reason for the antigenic difference between NHRI-RG3 and -RG6.

Because squalene-based adjuvant (AddaVax) can improve immune response and antibody cross-reactivity [28], we evaluated the immune response of alum- and AddaVax-adjuvanted H7N9 vaccines. The antisera of immunized mice were analyzed using an HI assay against homologous and heterologous H7N9 viruses. In the alum-adjuvanted groups, the geometric mean $\mathrm{HI}$ titers of NHRI-RG3, NHRI-RG4, NHRI-RG5, and NHRIRG6 vaccines against the homologous virus were 71.3, 80.0, 226.3, and 88.3, respectively (Fig. 4), and the NHRI-RG3, -RG5 and -RG6 vaccines induced a similar level of antibodies against homologous and heterologous H7N9 viruses (Fig. 4A, C, and D). However, the NHRIRG4 vaccine elicited statistically lower cross-reactive antibody (2- to 4-fold lower) against heterologous H7N9 viruses (NHRI-RG3, RG5 and RG6) than homologous H7N9 virus (NHRI-RG4) (Fig. 4B). In the AddaVaxadjuvanted groups, the geometric mean HI titers of NHRI-RG3, NHRI-RG4, NHRI-RG5, and NHRI-RG6 vaccines against the homologous virus were 127.0, 285.1, 285.1, and 160.0, respectively (Fig. 4). Compared with alum adjuvant, the AddaVax adjuvant slightly enhanced the immunogenicity of the NHRI-RG3, NHRI-RG5, and NHRI-RG6 vaccines (Fig. 4A, C, and D) and significantly improved the immunogenicity of the NHRI-RG4 vaccine against both homologous and heterologous viruses (Fig. 4B). These results indicate that NHRI-RG5 has the highest immunogenicity among all H7N9 CVVs, and the low immunogenicity and cross-reactivity of NHRI-RG4 vaccine may be improved using the AddaVax adjuvant.

\section{Discussion}

The manufacturing of vaccines for emerging pandemic influenza usually takes months with multiple processes, including the generation of high-growth CVVs, viral growth optimization, the evaluation of antigenicity and pathogenicity, and so on [26, 29, 30]. However, our experiences show that the use of egg-derived CVVs in cellbased manufacturing systems could prolong the time to final vaccine production because of the paperwork associated with CVV importation and the viral adaptation in 


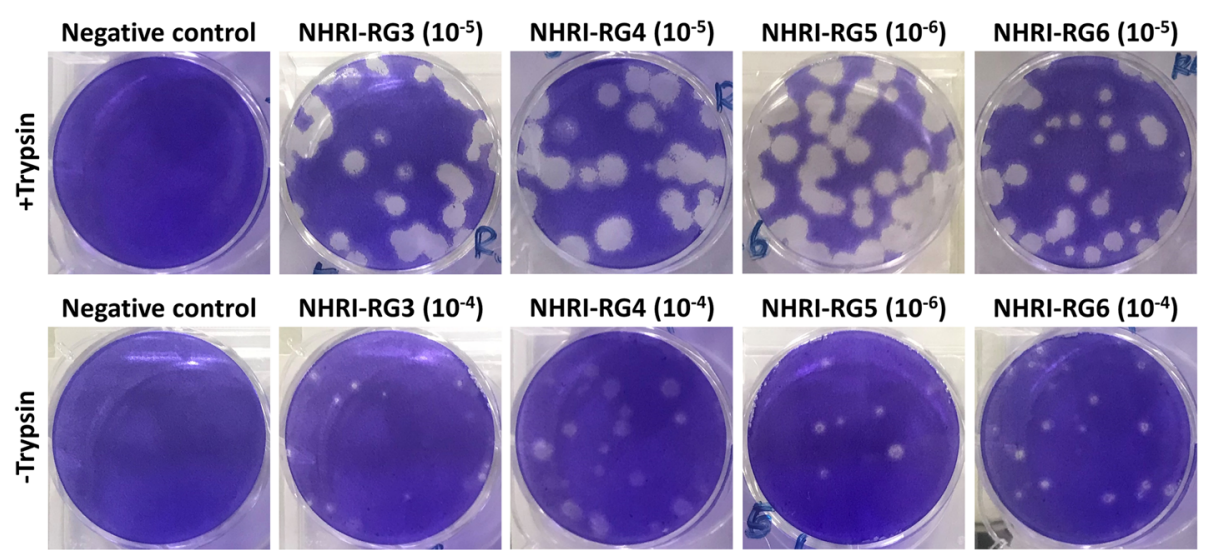

Fig. 3 Plaque-forming ability of H7N9 reassortant viruses in MDCK cells with or without trypsin. The indicated viruses were diluted to the value shown in parentheses and MDCK cells were inoculated with the diluted viruses. The negative control indicates MDCK cells treated with DPBS

cells (Fig. 1A). These processes may take an additional 2-4 months. In this study, we developed cell-derived CVVs using reverse genetics and generated high-yield CVVs in the sMDCK-manufacturing system (Fig. 1B). This approach could help save time by omitting the time of CVV importation and the optimization of viral growth in cells. Although a 3-passage adaptation to aMDCK cells was required to optimize the growth of NHRI-RG3 and NHRI-RG4 CVVs (Table 2), our results showed that the direct inoculation of the rescued reassortant virus in sMDCK cells improves virus growth without requiring further adaptation (right part in Fig. 1B).

Several suspension MDCK cell lines have also been developed to facilitate cell-based manufacturing processes [31-33]. Similar to these studies, the productivity of influenza viruses in SMDCK cells was higher than that in aMDCK cells (Fig. 2). The differences between sMDCK and aMDCK culture systems include cultivation conditions and the characteristics of the cell line. We found that the cell-specific productivity of viral particles (virions/cell) in the sMDCK culture system was 1.9- to 3.5fold higher than that in the aMDCK culture system (Supplementary Table S2). A previous study also showed that the HA production capability (HAU/ $10^{6}$ cells) of suspension MDCK cells is remarkably higher than that

Table 5 Biosafety assessment of H7N9 CWs

\begin{tabular}{ll}
\hline CWs & CELD $_{\mathbf{5 0}}{ }^{\mathrm{a}}$ \\
\hline Wild-type H7N9 (A/Taiwan/1/2017) & $10^{-1.17} \mathrm{TCID}_{50}$ \\
NHRI-RG3 & $>10^{6.65} \mathrm{TCID}_{50}$ \\
NHRI-RG4 & $>10^{7.04} \mathrm{TCID}_{50}$ \\
NHRI-RG5 & $>10^{7.65} \mathrm{TCID}_{50}$ \\
NHRI-RG6 & $>10^{6.65} \mathrm{TCID}_{50}$ \\
\hline
\end{tabular}

Note: ${ }^{\mathrm{a}} \mathrm{CELD} \mathrm{D}_{50}$ : median chicken embryo lethal dose; expressed as median tissue culture infectious dose $\left(\mathrm{TCID}_{50}\right)$ of adherent MDCK cells [31]. Therefore, we assume that the high yield of influenza virus in the sMDCK culture system does not depend entirely on cell density. To further explore the potential mechanism by which influenza virus growth is increased in sMDCK cells, we will examine the transcriptome differences between sMDCK and aMDCK cells.

Previous studies showed that $\mathrm{N}$-linked glycosylation at N123 and N149 sites of the H7 protein enhances the viral growth, which may be related to the influence on receptor binding [34, 35]. Similarly, we found that CVVs (NHRIRG5 and NHRI-RG6) with these N-linked glycosylation sites had 4-fold higher HA titers than NHRI-RG3 and NHRI-RG4 at passages V1aM1 and V1aM2 (Table 2). In particular, the HA titers of NHRI-RG3 and NHRI-RG4 at passage V1aM3 reached levels similar to those of NHRIRG5 and NHRI-RG6, which also have amino acid substitutions in the HA protein, including an I120T mutation in NHRI-RG3 and an A151T mutation in NHRI-RG4. The HA-I120T and HA-A151T mutations could potentially induce N-linked glycosylation of the HA protein at N118 (which is the same as NHRI-RG6) and N149 (which is the same as NHRI-RG5), respectively. The N-linked glycosylation at N118 (NHRI-RG3 and NHRI-RG6 and N149 (NHRI-RG4 and NHRI-RG5) in the HA protein has been shown by HPLC-MS/MS (Additional file 3: Fig. S2 and Additional file 4: Fig. S3). Based on previous studies [34, 35] and our findings, the acquired N-glycosylation site at N118 and N149 may be the reason for the improved HA titer of NHRI-RG3 and NHRI-RG4 (V1aM3), respectively, and these N-linked glycosylations at N118 and N149 did not cause any antigenic change in NHRI-RG3 and NHRI-RG4 (Table 6). Notably, the S118N mutation in the HA protein occurred gradually during evolution and became dominant in the 4th and 5th wave H7N9 viruses isolated from human, avian and environmental samples (Additional file 5: Fig. S4A), but the I120T mutation in the HA protein was found only in human 
Table $6 \mathrm{HI}$ activity of sheep standard serum against inactivated H7N9 bulks

\begin{tabular}{ll}
\hline CWs & HI titer \\
\hline Standard antigen (No. 18/196) & 640 \\
NHRI-RG3 & 640 \\
NHRI-RG4 & 640 \\
NHRI-RG5 & 640 \\
NHRI-RG6 & 160 \\
\hline
\end{tabular}

Note: Inactivated H7N9 bulks were prepared in sMDCK cells (V1aM3sM1)

samples (Additional file 5: Fig. S4B). Therefore, it is possible that N-linked glycosylation at N118 and N149 increases the growth efficiency of the H7N9 virus in mammalian cells. In the future, it could be an alternative to generate high-growth CVVs for emerging influenza H7N9 virus via introducing HA-I120T or HA-A151T substitutions, because it is acceptable to introduce special amino acid into the HA protein of influenza CVVs without affecting its antigenicity and pathogenicity [36].

Based on genetic and antigenic stability of influenza viruses, a suspension MDCK cell line, MDCK33016PF, has been considered as a suitable platform for the isolation and preparation of influenza vaccine seed viruses, especially H3N2 and B/Yamagata viruses [37]. The sequencing results of eight segments from NHRI-RG5 and -RG6 was quite stable in sMDCK cells after at least serial 7 passages, but several adaptive mutations, I120T, K163R, A292T, I421V, and I421M, were found in NHRIRG3 and -RG4 after serial 3 passages and required for improving growth efficiency. Although the role of each amino acid substitution in HA remains unclear, these results suggested that the genetic stability of H7N9 CVVs in sMDCK cells is strain-dependent. Further study is still required to explore the genetic and antigenic stability of various influenza viruses after serial passages in sMDCK cells and the potential of SMDCK cells to isolate and prepare influenza vaccine seed viruses.

Based on the WHO guideline to develop and produce pandemic influenza vaccines [26], this study conducted strict safety testing procedures to prove the pathogenicity of developed CVVs, including genetic stability, trypsin-independent plaque-forming ability, and biosafety evaluation. This study revealed that sMDCKderived high-growth H7N9 CVVs were attenuated with low levels of pathogenicity. Previous study using chicken

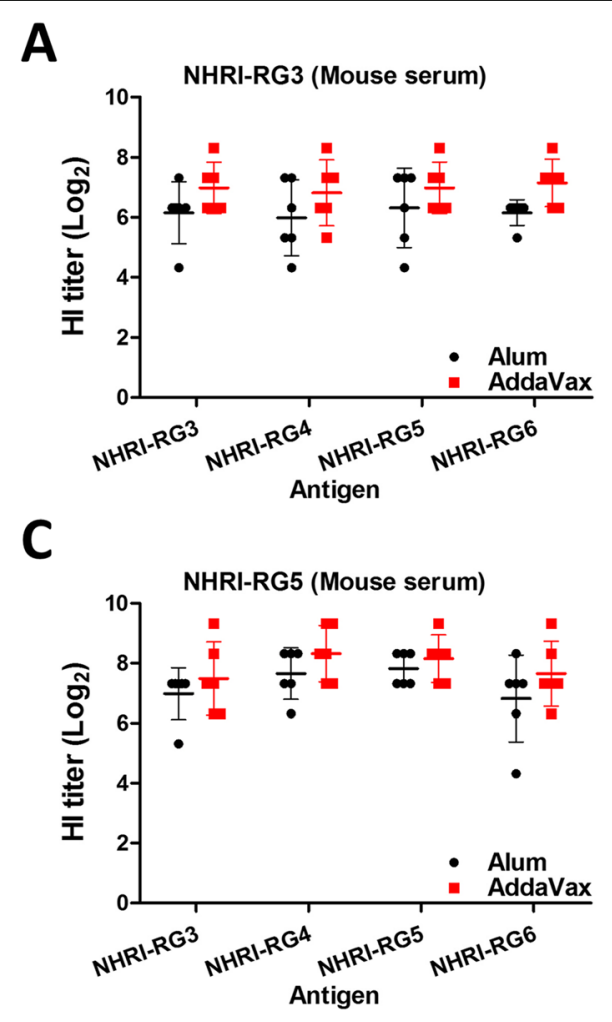

B

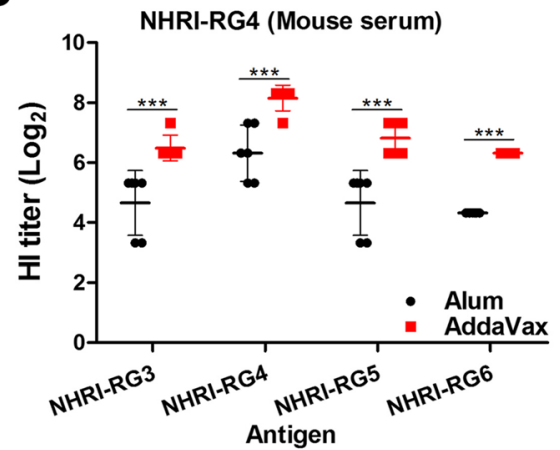

D

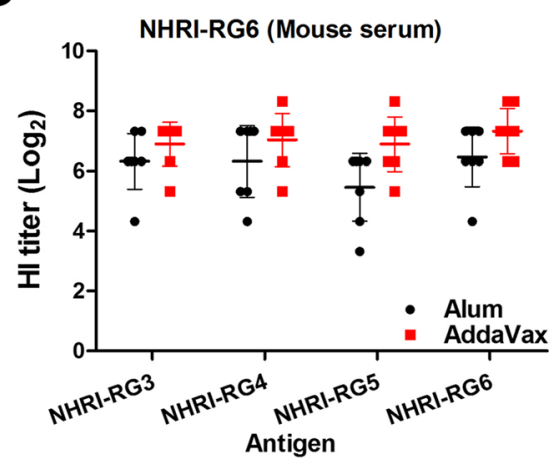

Fig. $4 \mathrm{HI}$ activity of mouse serum against inactivated H7N9 CWs. Each H7N9 bulk (NHRI-RG3, NHRI-RG4, NHRI-RG5, and NHRI-RG6) was mixed with alum or AddaVax adjuvant, and administered to BALB/c mice intramuscularly on day 0 and day 14 . The reactivity of mouse serum against inactivated NHRI-RG3, NHRI-RG4, NHRI-RG5, and NHRI-RG6 CWs (Antigen) was examined by an HI assay, using the serum collected on day 28. The line indicates the geometric mean with a $95 \%$ confidence interval. The $\log _{2}$-transformed $\mathrm{HI}$ titers were analyzed by ANOVA with Newman-Keuls post-test ( $n=6$ mice for NHRI-RG3, NHRI-RG4 and NHRI-RG5 groups; $n=7$ mice for NHRI-RG6 group). Significant differences between alum- and AddaVax-adjuvanted vaccines are indicated by ${ }^{* *}, p<0.001$ 
embryos lethality test showed that reassortant H5N1 CVVs lacks pathogenicity compared with wild-type HPAI viruses, which are consistent with the safety test in ferrets and chickens [27]. According the $\mathrm{WHO}$ guideline [26, 38], the safety study should be conducted in ferrets before the CVVs are released to vaccine manufacturers with BSL-2 enhanced containment. This study aims to develop high-growth influenza CVVs in suspension MDCK cells before vaccine manufacturing; hence, we will conduct ferret study in the future before the CVVs are used as vaccine seed viruses.

Although a WHO report showed that the antigenicity of LPAI H7N9 viruses is distinct from that of emerging HPAI H7N9 viruses [39], a previous study illustrated that the AddaVax- adjuvanted LPAI H7N9 vaccine conferred efficient protection against HPAI H7N9 virus infection in a ferret challenge model [40]. Consistently, we found that alum-adjuvanted NHRI-RG4 (LPAI) vaccine induced antibodies with low cross-reactivity against HPAI H7N9 viruses (NHRI-RG3, NHRI-RG5, and NHRI-RG6), but this low cross-reactivity was improved by the use of the AddaVax adjuvant, an MF-59-like adjuvant (Fig. 4B). This improvement has been reported in MF-59-adjuvanted H1N1 vaccines [41]. Interestingly, the antigenicity of NHRI-RG6 was distinct from A/Guangdong/17SF003/2016-like antigen (Table 6), but alum- and AddaVax- adjuvanted HPAI H7N9 vaccines (NHRI-RG3, NHRI-RG5, and NHRI-RG6) could elicit high cross-reactive antibody response to each other (Fig. 4). We also found that AddaVax adjuvant inclusion enhanced the immunogenicity of NHRI-RG4 (Fig. 4B), consistent with the dose-sparing effect of AddaVax adjuvant on the H7N9 vaccine in mice [28]. These findings demonstrate that squalene-based adjuvants have the potential to improve H7N9 vaccine efficacy by increasing crossreactivity and immunogenicity.

\section{Conclusions}

In summary, we used 6 internal genes of an aMDCK celladapted MDV and synthetic HA and NA genes to generate influenza H7N9 reassortant vaccine viruses by establishing reverse genetics. We further found that these cell-derived CVVs have high growth rates in SMDCK cells and demonstrated that four cell-derived H7N9 CVVs have a trypsindependent plaque-forming ability and no lethality in chicken embryos. To shorten the preparation time of CVVs for production, it might be ideal to directly establish SMDCK cellderived CVVs, which could improve the process of CVV preparation by eliminating the use of aMDCK cells. Moreover, the comparison of immunogenic and antigenic properties among the four H7N9 CVVs showed that NHRI-RG5 is the most suitable for the production of prepandemic vaccines. In conclusion, combining the two plaforms (sMDCK and reverse genetics) could significantly improve efficiency and productivity of manufacturing influenza H7N9 vaccines for pandemic preparedness.

\section{Supplementary information}

Supplementary information accompanies this paper at https://doi.org/10. 1186/s12929-020-00645-y.

Additional file 1: Table S1. Major differences in the HA1 amino acid sequence of selected reassortant H7N9 viruses.

Additional file 2: Table S2. Cell-specific productivity of MDCK cells. Additional file 3: Fig. S1. EM images of H7N9 bulks. sMDCK-derived H7N9 reassortant viruses were purified, viral particles were negatively stained with $2 \%$ UA, and the images were captured using EM.

Additional file 4: Fig. S2. Identification of N-linked glycosylation at the $\mathrm{N} 118$ residue on hemagglutinin by liquid chromatography-tandem mass spectrometry. N-linked glycosylation was identified by liquid chromatography-tandem mass spectrometry, as described in Additional file 6. Tandem mass spectra (MS2) of ESGGIDKEPMGFTYNGTR (m/z $653.96,+3$ ) derived from the trypsin-digested purified H7N9 bulks, (A) NHRI-RG3 and (B) NHRI-RG6. N\# represents the deamidated asparagine which indicates that the $\mathrm{N} 118$ residue is glycosylated in the original hemagglutinin protein.

Additional file 5: Fig. S3. Identification of N-linked glycosylation at the N149 residue on hemagglutinin by liquid chromatography-tandem mass spectrometry. $\mathrm{N}$-linked glycosylation was identified by liquid

chromatography-tandem mass spectrometry, as described in Additional file 6. Tandem mass spectra (MS2) of WLLSNTDNATFPQMTK (m/z 934.44, + 2) derived from the trypsin-digested purified H7N9 bulks, (A) NHRI-RG4 and (B) NHRI-RG5. N\# represents the deamidated asparagine which indicates that the $\mathrm{N} 149$ residue is glycosylated in the original hemagglutinin protein.

Additional file 6: Fig. S4. Evolution of the hemagglutinin N118 glycosylation site in H7N9 viruses from the 1st to 5th epidemic wave. Temporal pattern of S118N (A) and I120T (B) mutations in H7N9 hemagglutinin from human, avian and environmental samples. HA protein sequences were collected and analyzed as described in Additional file 6.

Additional file 7: Supplemental materials and methods

\section{Abbreviations}

aMDCK: Adherent MDCK cells; CWs: Candidate vaccine viruses; CELD $_{50}$ : Median chicken embryo lethal dose; E: Eggs; HPAl: Highly pathogenic avian influenza; HA: Hemagglutinin; HAU: Hemagglutination units; LPAl: Low pathogenic avian influenza; MDVs: Master donor viruses; NA: Neuraminidase; V: Vero cells; DPI: Day post infection; $\mathrm{TCID}_{50}$ : Median tissue culture infectious dose; sMDCK: Suspension MDCK cells

\section{Acknowledgments}

We would like to acknowledge team members from the ABSL-3 laboratory of bioproduction plant at the NHRI, especially Ms. Jung-Shu Weng and Mr. Cheng-Han Hsieh. We gratefully acknowledge Dr. Shang-Rung Wu at the Instrument Development Center of the National Cheng Kung University, Taiwan, for the work of TEM images. We also gratefully acknowledge the authors, originating and submitting laboratories of the sequences from GISAID's EpiFlu $^{\text {TM }}$ Database used in sequence analysis and CWs selection.

\section{Authors' contributions}

TTT, PLC, WCS, MSL, and AYH were involved in the conception and the design of the studies. TTT, PLC, TCW, SYT, and HIC performed the experiments and acquired the data. CC Lai, PWC, CC Lu, helped purify the inactivated viruses. TाT wrote the manuscript with input from all authors and PLC, MTL coedited the paper. All authors have read and approved the final version of the manuscript.

\section{Funding}

Funding source is providing from the National Flagship Project in Taiwan (MOST 106-3114-Y404-002; 107-0324-01-19-13).

\section{Availability of data and materials}

The data that support the findings of this study are available from the corresponding author upon request. 


\section{Ethics approval}

The mouse study protocol was approved by the Institutional Animal Care and Use Committee of NHRI (Number of Permission: NHRI-IACUC-107106-A).

\section{Consent for publication}

Not applicable.

\section{Competing interests}

The authors declare no competing interests.

\section{Author details}

${ }^{1}$ National Institute of Infectious Diseases and Vaccinology, National Health Research Institutes (NHRI), 35 Keyan Road, Zhunan, Miaoli County 35053, Taiwan. ${ }^{2}$ Institute of Molecular and Cellular Biology, National Tsing Hua University, Hsinchu, Taiwan. ${ }^{3}$ College of Life Science, National Tsing Hua University, Hsinchu, Taiwan. ${ }^{4}$ Centers for Disease Control, Ministry of Health and Welfare, Taipei 689, Taiwan.

Received: 16 September 2019 Accepted: 27 March 2020

Published online: 02 April 2020

\section{References}

1. Food and Agriculture Organization. http://www.fao.org/ag/againfo/ programmes/en/empres/h7n9/situation_update.html. Accessed 27 Jun 2019.

2. Zhu W, Zhou J, Li Z, Yang L, Li X, Huang W, et al. Biological characterisation of the emerged highly pathogenic avian influenza (HPAl) A(H7N9) viruses in humans, in mainland China, 2016 to 2017. Euro Surveill. 2017;22(19):30533.

3. Yang JR, Liu MT. Human infection caused by an avian influenza a (H7N9) virus with a polybasic cleavage site in Taiwan, 2017. J Formos Med Assoc. 2017;116(3):210-2.

4. Liu X, Li T, Zheng Y, Wong KW, Lu S, Lu H. Poor responses to oseltamivir treatment in a patient with influenza a (H7N9) virus infection. Emerg Microbes Infect. 2013;2(5):e27

5. Hu Y, Lu S, Song Z, Wang W, Hao P, Li J, et al. Association between adverse clinical outcome in human disease caused by novel influenza a H7N9 virus and sustained viral shedding and emergence of antiviral resistance. Lancet. 2013;381(9885):2273-9.

6. Lee MS, Hu AY. A cell-based backup to speed up pandemic influenza vaccine production. Trends Microbiol. 2012;20(3):103-5.

7. Hegde NR. Cell culture-based influenza vaccines: a necessary and indispensable investment for the future. Hum Vaccin Immunother. 2015;11(5):1223-34.

8. Harding AT, Heaton NS. Efforts to Improve the Seasonal Influenza Vaccine. Vaccines. 2018;6(2):19-31

9. Murakami S, Horimoto T, Mai le Q, Nidom CA, Chen H, Muramoto Y, et al. Growth determinants for $\mathrm{H} 5 \mathrm{~N} 1$ influenza vaccine seed viruses in MDCK cells. J Virol. 2008:82(21):10502-9.

10. Tseng YF, Hu AY, Huang ML, Yeh WZ, Weng TC, Chen YS, et al. Adaptation of high-growth influenza H5N1 vaccine virus in Vero cells: implications for pandemic preparedness. PLoS One. 2011;6(10):e24057.

11. Hu AY, Tseng YF, Weng TC, Liao CC, Wu J, Chou AH, et al. Production of inactivated influenza $\mathrm{H} 5 \mathrm{~N} 1$ vaccines from MDCK cells in serum-free medium. PLoS One. 2011;6(1):e14578.

12. Chia MY, Hu AY, Tseng YF, Weng TC, Lai CC, Lin JY, et al. Evaluation of MDCK cellderived influenza H7N9 vaccine candidates in ferrets. PLoS One. 2015;10(3):e0120793.

13. Ping J, Lopes TJS, Nidom CA, Ghedin E, Macken CA, Fitch A, et al. Development of high-yield influenza a virus vaccine viruses. Nat Commun. 2015;6:8148.

14. Kim EH, Kwon HI, Park SJ, Kim YI, Si YJ, Lee IW, et al. Generation of a highgrowth influenza vaccine strain in MDCK cells for vaccine preparedness. J Microbiol Biotechnol. 2018;28(6):997-1006.

15. Hu W, Zhang H, Han Q, Li L, Chen Y, Xia N, et al. A Vero-cell-adapted vaccine donor strain of influenza a virus generated by serial passages. Vaccine. 2015;33(2):374-81

16. World Health Organization. https://www.who.int/influenza/vaccines/virus/ candidates_reagents/summary_a_h7n9_cw_20180305.pdf. Accessed 22 Mar 2018

17. Dormitzer PR, Suphaphiphat P, Gibson DG, Wentworth DE, Stockwell TB, Algire MA, et al. Synthetic generation of influenza vaccine viruses for rapid response to pandemics. Sci Transl Med. 2013;5(185):185ra168.

18. Tang J, Zhang J, Zhou J, Zhu W, Yang L, Zou S, et al. Highly pathogenic avian influenza H7N9 viruses with reduced susceptibility to neuraminidase inhibitors showed comparable replication capacity to their sensitive counterparts. Virol J. 2019;16(1):87.

19. Hoffmann E Stech J, Guan Y Webster RG, Perez DR. Universal primer set for the fulllength amplification of all influenza a viruses. Arch Virol. 2001;146(12):2275-89.
20. Hoffmann E, Neumann G, Kawaoka Y, Hobom G, Webster RG. A DNA transfection system for generation of influenza a virus from eight plasmids. Proc Natl Acad Sci U S A. 2000;97(11):6108-13.

21. REED $L$, MUENCH H. A simple method of estimating fifty percent endpoints. Am J Epidemiol. 1938;27(3):493-7.

22. Tseng YF, Weng TC, Lai CC, Chen PL, Lee MS, Hu AY. A fast and efficient purification platform for cell-based influenza viruses by flow-through chromatography. Vaccine. 2018;36(22):3146-52.

23. World Health Organization. https://www.who.int/biologicals/areas/vaccines/ TRS_979_Annex_5.pdf. Accessed 5 Jun 2019.

24. World Health Organization. https://www.who.int/csr/resources/publications/ influenza/whocdscsrncs20025rev.pdf. Accessed 1 Mar 2017.

25. Benne CA, Kroon FP, Harmsen M, Tavares L, Kraaijeveld CA, De Jong JC. Comparison of neutralizing and hemagglutination-inhibiting antibody responses to influenza a virus vaccination of human immunodeficiency virus-infected individuals. Clin Diagn Lab Immunol. 1998;5(1):114-7.

26. World Health Organization. https://www.who.int/influenza/resources/ documents/influenza_vaccine-virus_selection/en/. Accessed 19 Nov 2018.

27. Dong J, Matsuoka Y, Maines TR, Swayne DE, O'Neill E, Davis CT, et al. Development of a new candidate H5N1 avian influenza virus for pre-pandemic vaccine production. Influenza Other Respir Viruses. 2009;3(6):287-95.

28. Wu CY, Chang CY, Ma HH, Wang CW, Chen YT, Hsiao PW, et al. Squaleneadjuvanted $\mathrm{H7N} 9$ virus vaccine induces robust humoral immune response against H7N9 and H7N7 viruses. Vaccine. 2014;32(35):4485-94.

29. World Health Organization. https://www.who.int/csr/disease/swineflu/notes/ h1n1_vaccine_20090806/en/. Accessed 17 Oct 2018.

30. Centers for Disease Control and Prevention. https://www.cdc.gov/flu/ avianflu/candidate-vaccine-virus.htm. Accessed 17 Jan 2018.

31. Chu C, Lugovtsev V, Golding H, Betenbaugh M, Shiloach J. Conversion of MDCK cell line to suspension culture by transfecting with human siat7e gene and its application for influenza virus production. Proc Natl Acad Sci U S A. 2009;106(35):14802-7.

32. Huang D, Peng WJ, Ye Q, Liu XP, Zhao L, Fan L, et al. Serum-free suspension culture of MDCK cells for production of influenza H1N1 vaccines. PLoS One. 2015;10(11):e0141686.

33. Lohr V, Genzel Y, Behrendt I, Scharfenberg K, Reichl U. A new MDCK suspension line cultivated in a fully defined medium in stirred-tank and wave bioreactor. Vaccine. 2010;28(38):6256-64

34. Chen Z, Baz M, Lu J, Paskel M, Santos C, Subbarao K, et al. Development of a high-yield live attenuated H7N9 influenza virus vaccine that provides protection against homologous and heterologous $\mathrm{H} 7$ wild-type viruses in ferrets. J Virol. 2014;88(12):7016-23.

35. Nakamura K, Shirakura M, Suzuki Y, Naito T, Fujisaki S, Tashiro M, et al. Development of a high-yield reassortant influenza vaccine virus derived from the a/Anhui/1/2013 (H7N9) strain. Vaccine. 2016:34(3):328-33.

36. Li X, Gao Y, Ye Z. A Single Amino Acid Substitution at Residue 218 of 786 Hemagglutinin Improves the Growth of Influenza A(H7N9) Candidate 787 Vaccine Viruses. J Virol. 2019;93(19):e00570-19.

37. Nakamura K, Harada Y, Takahashi $\mathrm{H}$, Trusheim H, Bernhard R, Hamamoto I, et al. Systematic evaluation of suspension MDCK cells, adherent MDCK cells, and LLCMK2 cells for preparing influenza vaccine seed virus. Vaccine. 2019;37(43):6526-34.

38. World Health Organization. https://www.who.int/biologicals/BIOSAFETY_ RISK_ASSESSMENT_21_MAY_2013.pdf. Accessed 17 Jan 2018.

39. World Health Organization. https://www.who.int/influenza/vaccines/virus/2 01703 zoonotic vaccinevirusupdate.pdf. Accessed 22 Mar 2018

40. Hatta M, Zhong G, Chiba S, Lopes TJS, Neumann G, Kawaoka Y. Effectiveness of whole, inactivated, low pathogenicity influenza a(H7N9) vaccine against Antigenically distinct, highly pathogenic H7N9 virus. Emerg Infect Dis. 2018;24(10):1910-3.

41. Khurana S, Verma N, Yewdell JW, Hilbert AK, Castellino F, Lattanzi M, et al. MF59 adjuvant enhances diversity and affinity of antibody-mediated immune response to pandemic influenza vaccines. Sci Transl Med. 2011;3(85):85ra48.

\section{Publisher's Note}

Springer Nature remains neutral with regard to jurisdictional claims in published maps and institutional affiliations. 